\title{
Frontières
}

\section{Du banal au sublime}

Célébrer la mort

\section{Raymond Lemieux}

Volume 18, numéro 1, automne 2005

Hélas, célébrer la mort!

URI : https://id.erudit.org/iderudit/1074308ar

DOI : https://doi.org/10.7202/1074308ar

Aller au sommaire du numéro

\section{Éditeur(s)}

Université du Québec à Montréal

ISSN

1180-3479 (imprimé)

1916-0976 (numérique)

Découvrir la revue

Citer cet article

Lemieux, R. (2005). Du banal au sublime : célébrer la mort. Frontières, 18(1), 8-15. https://doi.org/10.7202/1074308ar
Résumé de l'article

On assiste aujourd'hui à une recomposition des rituels entourant la mort, ceux-ci passant d'une gestion religieuse traditionnelle, soutenue par les communautés confessionnelles, vers une gestion marchande. Cette recomposition suscite l'apparition de nouveaux rites, de même qu'une redéfinition des fonctions rituelles. Après avoir posé l'importance de ces dernières d'un point de vue anthropologique, l'auteur tente d'identifier et de qualifier certaines de leurs transformations contemporaines, réparties en trois niveaux structurels que représentent l'acte de mourir, la disposition des restes et les croyances en l'après-vie. Comment s'y effectue désormais le travail de deuil, dans chacun de ces niveaux et comment tente de s'y recomposer un rapport organique à la communauté humaine?
Ce document est protégé par la loi sur le droit d'auteur. L'utilisation des services d'Érudit (y compris la reproduction) est assujettie à sa politique d'utilisation que vous pouvez consulter en ligne.

https://apropos.erudit.org/fr/usagers/politique-dutilisation/ 
Résumé

On assiste aujourd'hui à une recomposition des rituels entourant la mort, ceux-ci passant d'une gestion religieuse traditionnelle, soutenue par les communautés confessionnelles, vers une gestion marchande. Cette recomposition suscite l'apparition de nouveaux rites, de même qu'une redéfinition des fonctions rituelles. Après avoir posé l'importance de ces dernières d'un point de vue anthropologique, l'auteur tente d'identifier et de qualifier certaines de leurs transformations contemporaines, réparties en trois niveaux structurels que représentent l'acte de mourir, la disposition des restes et les croyances en l'après-vie. Comment $s^{\prime} y$ effectue désormais le travail de deuil, dans chacun de ces niveaux et comment tente de s'y recomposer un rapport organique à la communauté humaine?

Mots clés: célébration - rites communauté - deuil.

\section{Abstract}

Rituals surrounding death are to-day in a reconstruction process, in consequence of the transformations in the social regulations of death passing from confessional and community rules to market ones. This reconstruction emerges as evidence in new secular forms of rituals, on the one hand, and in a redefinition of ritual functions, on the other. After having stressed the importance of anthropological general functions of rituals, the paper identifies and qualifies some contemporary transformations of death rituals, distributing them in three structural levels: the act of dying, the disposal of remains and the after-life imaginary. How the act of grieving for the lost of a life does now come to the symbolic order, and what do mean the new links to be connected with the human community?

Keywords: celebration - rites community-mourning.

\section{DU BANAL AU SUBLIME CÉLÉBRER LA MORT}

\section{ENTRE LES RIVES DU MÊME ET DE L'AUTRE, L'HOMME EST UN PONT.}

JEAN-PIERRE VERNANT ${ }^{1}$

\section{Raymond Lemieux, Ph.D., \\ professeur, Faculté de théologie et de sciences religieuses, Université Laval.}

Le décès d'un être humain n'est pas seulement la fin d'une existence, c'est un événement social qui représente l'irruption d'un insensé dans l'ordre du sens. Voilà sans doute pourquoi, dans nos sociétés, la mort est marquée d'une symbolisation paradoxale: d'une part, elle est objet d'une surexploitation médiatique, d'autre part, elle est réduite à l'intime et au privé. Virtuelle, mort de l'autre, de l'étranger, elle fait la une des médias dans l'ostentation de sa violence; réelle, mort d'un proche ou approche de sa propre mort, elle fait objet d'une trouble pudeur, comme si elle portait en elle une part de l'humain à la limite de l'avouable, quasi obscène.

Nous passons notre vie à inventer du sens, à travailler à ce que la suite de nos jours puisse représenter un projet, voire un progrès par rapport aux générations qui nous ont précédés. Nous nous acharnons à croire en ce progrès pour le faire advenir, parce que sans progrès, il n'y aurait pas d'histoire humaine plausible. Or c'est précisément ce travail du croire qui installe l'humain dans l'ordre du sens, exigeant de lui de constants efforts de dépassement, dans des tentatives toujours risquées de traversée de l'insensé. C'est précisément cela que la mort met en cause. Elle en contredit les acquis, par sa violence propre, et en impose, du même coup, le recommencement.
Les institutions humaines, qui ont toutes à voir avec cette forme primaire d'institution qu'est le sens, sont donc une forme de pari contre la mort, pari qui ne saurait être tenu, sauf à être constamment renouvelé. La mort introduit un trauma dans l'ordre symbolique, trauma qui se traduit par une mise en balance de la réalité du sujet et de ses objets. Dès lors, célébrer la mort, quel que soit le rituel impliqué, apparaît une pure absurdité, puisque cela revient à célébrer par des signes l'insignifiance de tout signe: qu'est-ce qu'un signe s'il n'y a plus personne pour l'interpréter? À moins de supposer que tout rituel constitue une forme de reconnaissance de la fragilité et du caractère à la fois transitoire et infondé du sens. C'est donc la notion même de rituel qu'il s'agit ici d'interroger. Cela permettra de mettre en lumière les transformations des rites funéraires occidentaux au cours du dernier demi-siècle, et de tenter de mieux comprendre dans quelle mesure l'imbroglio rituel contemporain s'insère dans une dynamique du sens beaucoup plus large, elle-même à placer dans une problématique globale de la survie qui semble toujours la clé des discours sur la mort. L'expérience de la mort impose un pari sur la vie. Ce pari, nous le verrons en bout de piste, n'est pas seulement celui d'une énigmatique vie après la mort - ce qui suppose d'entrer dans l'ordre du croire et se situe hors de notre propos - mais celui de la vie elle-même, dans sa continuité, malgré l'insensé de l'expérience de la mort qui, par ailleurs, en est partie prenante. 


\section{LES RITUELS: UNE PRATIQUE}

\section{DES LIMITES}

Un ami ayant vécu plusieurs années en Inde s'amusait à poser à son entourage québécois la blague suivante: "Qu'est-ce qui s'oppose à la mort?» Immanquablement, en bons Occidentaux, nous répondions: «La vie». «Eh bien non, aimait-il rétorquer, si vous aviez vécu en Inde, vous sauriez que ce qui s'oppose à la mort, c'est la naissance. La naissance et la mort font partie de la vie...».

La vie est faite de limites, malgré les utopies et les constructions du désir qui cherchent à en nier le fait. La conscience qui l'habite n'est que la fragile et éphémère construction d'une consistance en creux du réel qui lui échappe, hors limites, réel sidéral et sidérant qui reste pour elle chaotique et représente - parce qu'il faut bien le représenter - l'envers même de l'ordre symbolique dont se prévalent ses constructions.

Dans ce contexte, l'objectivité de nos identités humaines est bien peu de chose. En fait, elle se donne essentiellement, elle aussi, par un repérage des limites: "Alexandre, Pella - 356/Babylone - 329», notent les dictionnaires de celui qui est également dit «le Grand », conquérant, bâtisseur de villes et tyran de l'Antiquité; "Alexandre, Játiva 1431 / Rome 1503 », notent-ils au sujet du sixième pape du nom, de la famille Borgia, intrigant, homme de pouvoir et fauteur de scandale dans son Église. Célèbre ou manant, chacun naît en un certain lieu, à un moment donné et meurt en un certain lieu, à un moment donné. L'objectivité d'une existence dans la mémoire collective ne tient quasiment qu'à cela seul, comme les dictionnaires en attestent, parce que seules ces données relèvent d'un ordre symbolique sur lequel tous s'entendent - la mesure du temps. Le reste est subjectif et fait appel à l'imaginaire, individuel ou collectif. Les qualités et défauts attribués aux personnages relèvent d'une mémoire historique tardive. Ils servent des intérêts étrangers au réel de leur existence passée, intérêts relevant des valeurs actuelles et non de celles de leur époque. En réalité, nous n'avons aucune idée des désirs profonds et des conflits de conscience qui ont pu agiter chacun des deux Alexandre, de leur vivant. En les soumettant à nos valeurs, nous pouvons cependant nous faire une idée de la violence des empires, que nous craignons, ou de la nature corruptible des institutions dont la crédibilité nous a cessé d'être évidente. Nous donnons de l'épaisseur au trait brut de leur vie qui se trace entre les deux dates, celle de leur naissance et celle de leur mort, point d'origine et point de rupture de cette possibilité du sens, devenue étrangère pour eux, mais actuelle pour nous.

\section{CÉLÉBRER LA MORT, QUEL QUE SOIT LE RITUEL IMPLIQUÉ,}

APPARAÎT UNE PURE ABSURDITÉ, PUISQUE CELA REVIENT

\section{À CÉLÉBRER PAR DES SIGNES L'INSIGNIFIANCE DE TOUT SIGNE:}

\section{QU'EST-CE QU'UN SIGNE S'IL N'Y A PLUS PERSONNE}

\section{POUR L'INTERPRÉTER?}

Autrement dit, la fin de la vie signifie, pour l'humain, que les jeux sont faits. La responsabilité du sens passe à quelqu'un d'autre. Sans doute cela représente-t-il le point focal du trauma. Dans l'expérience de la finitude, chacun bute sur les bornes de son histoire. Un point de bascule s'impose dans l'ordre du sens dont se constitue la vie. Quel que soit le contrôle que nous pensons exercer sur l'ordre tant que dure la vie, nous savons que la responsabilité du sens va nous échapper quand se produira l'inéluctable, puisque cette responsabilité nous échoit déjà, à notre corps défendant, quand c'est un autre qui disparaît. La mort signifie que notre histoire est, en dernière analyse, hétéronome.

Tant qu'on vit, on peut bien penser contribuer à la construction de ce qui va rester de cette vie, à produire sa consistance, à la suggérer, voire à l'imposer aux autres. Le sens, alors, relève encore de la responsabilité du sujet et nulle part ne s'articule mieux que dans ses rêves d'autonomie, si l'autonomie réside bien dans «la capacité, d'une société ou d'un individu, d'agir délibérément et explicitement pour modifier sa loi, c'est-à-dire sa forme ${ }^{2} »$. L'autonomie suppose une éthique de l'existence qui consiste, précisément, dans ce mode selon lequel la subjectivité est concernée par l'ordre du sens. Mais le «disparu» ne peut plus contribuer à cette production de sens. L'épaisseur de sa vie, ce qui donne consistance à la ligne tracée entre sa naissance et sa mort, à son nom, va désormais appartenir aux autres. Bien entendu, le disparu va encore influencer, pour un temps, les représentations que les autres entretiendront de lui. Celles-ci vont être soutenues par ce qu'on estime avoir été sa volonté, à travers la mémoire plus ou moins éphémère qui la rappellera. Mais sa participation à la culture - l'ensemble des échanges soutenus par les «valeurs» sur lesquels on s'entend va se faire sans lui.

Or c'est précisément là, au cœur de cette dynamique, dans ce «cœur déchiré de l'existence ${ }^{3}$ », que se pose la question des rituels.

Pour l'anthropologue Victor Turner ${ }^{4}$, qui en a traqué les multiples formes, le rituel est une expérience liminale, du mot latin limen, seuil. Ses mises en scène consistent essentiellement à travailler le sens là où se manifestent les limites de la conscience humaine. Si la mort représente un point de bascule dans l'ordre du sens, comme nous en convainc l'expérience commune, elle appelle tout naturellement la ritualisation. Celle-ci vise à reconstituer l'harmonie brisée, avec soi-même, avec les autres et avec le cosmos, à recomposer les idéaux de la conscience malgré l'incohérence du vécu, et, en dernière analyse, à jeter des ponts sur l'angoisse suscitée par cette incohérence. Elle propose pour cela de revisiter les frontières de cette conscience, alors même que l'irruption de l'altérité l'a bouleversée. Elle le fait cependant à la manière d'un voyage organisé, à travers des codes établis. Et par là, la ritualisation laisse entendre qu'une certaine domestication de l'Autre est plausible. Elle transforme le chaos en harmonie, l'horreur en beauté, le tourment en paix, bref, elle tente d'intégrer quelque chose de l'Autre dans la vie, malgré le trauma, pour montrer que, malgré tout, le sens reste possible. Le rituel visite ainsi les bords de l'intouchable - non-lieu ou sacré - en les faisant venir au langage, pour les apprivoiser, un langage où le réel reste certes au-delà, mais où quelque chose peut aussi sembler en être maîtrisé. Dès lors sur ces plages de la conscience mise à vif, l'Autre plutôt que sauvage, peut se faire caressant. On peut l'attendre, espérant qu'il vienne baiser ces bords dont la vie est faite, dans une mise en scène qui n'est jamais sans érotisme, puisque chacun sait bien qu'elle peut lui être fatale s'il s'y abandonne complètement, mais qu'elle est, ô combien, vivifiante tant qu'il la maîtrise.

En termes psychanalytiques, on pourrait dire que le rituel vise à domestiquer la jouissance de l'Autre. On comprend dès lors que, pour être efficace, il doive en reprendre les termes, mais dans un mode apprivoisé. Le rituel fait de l'Autre sauvage le collaborateur d'une histoire sensée. Là où s'imposent la rupture et le chaos, il prétend restaurer les trames déchirées du tissu social. Pour Victor Turner, son enjeu représente rien de moins que la possibilité de la communitas face à la societas. Selon ses termes, la «communauté» représente ici 
une expérience d'entente quant au sens, à la solidarité humaine, à l'harmonie dans la convivialité, alors que la «société» désigne l'organisation sociale objective, l'expérience d'être séparé des autres, de soi-même et du cosmos par les structures sociales. Sur les traces de Turner, une autre anthropologue, Catherine Bell, pousse cette logique encore plus loin et cherche dans le rituel non seulement une fonction d'intégration (aux communautés existantes) mais un pouvoir intégratif ${ }^{5}$, c'est-à-dire la capacité de valoriser les manifestations de la différence, voire le chaos lui-même, pour les intégrer dans l'harmonie.

Particulièrement étudié par Victor et Edith Turner, le pèlerinage représente une sorte de prototype $^{6}$ de ce travail $d u$ rituel. En effet, le voyage ritualisé suppose un retrait provisoire de la vie quotidienne pour mieux la réintégrer ensuite. Le pèlerin expérimente dans ce voyage les limites de sa vie habituelle. Il prend le risque de l'étranger, l'autre qu'il faut apprendre à connaître, les pays inconnus qu'il faut traverser, les mœurs inhabituelles qu'il faut apprivoiser, et, au bout de sa route, avant son retour, il espère trouver l'objet de sa quête, un Autre transcendant et utopique, qui restera toujours différent de l'image qu'il s'en est faite mais qui aura dès lors marqué son existence. Il en conservera précieusement quelques objets-traces (coquilles de Compostelle, eau des sources miraculeuses, cailloux du désert) qui rappelleront, à l'usage du quotidien, l'expérience de cette rencontre de la limite.

La communauté rituelle, avancent encore les Turner dans cette analyse, se situe "en rupture avec l'espace de sociabilité ordinaire ${ }^{7}$. Le pèlerin suspend la routine de sa vie quotidienne "au bénéfice d'une sociabilité plus "horizontale", plus "fraternelle", voire plus fusionnelle ${ }^{8}$ ". Non régie par les règles coutumières de sa culture, déployée dans un autre environnement, elle est transgressive. Et cette transgression (au sens premier du mot: marcher à travers, traverser), passage vers un autre espace (ou vers l'espace de l'Autre) permet au sujet qui s'y risque de se réapproprier du sens oublié ou empoussiéré dans la quotidienneté. L'expérience de l'Autre, dans le rite, renvoie au même. Elle est faite pour le régénérer, lui donner un nouveau souffle. Il arrive aussi qu'elle le transforme après l'avoir nourri.

Il va sans dire qu'ainsi considérés les rituels n'appartiennent pas seulement à cette dimension de la culture qu'on appelle «religieuse» et dont la fonction spécifique consiste précisément à inscrire dans la vie sociale la dimension transcendantale de l'expérience de l'Autre. Les institutions religieuses proposent des modalités particu- lières de ritualisation accordées à une certaine entente quant au sens, telle qu'elle est retenue par leur tradition. Elles appellent leurs fidèles à s'entendre à leur tour, à partir de là, pour gérer leur propre rapport au sens, dans l'accueil de cette transcendance et la prise en compte des conséquences pratiques dans leurs manières de vivre. Il est évident pour cela qu'aucune religion ne saurait se passer de rituel. Mais d'autres types d'expérience liminale peuvent aussi s'avérer des lieux possibles de régénération de l'humain: le théâtre, le voyage, la prise de contact avec la nature, voire certaines expériences de divertissement. Il existe aujourd'hui sans doute davantage de rituels séculiers, en congruence par rapport à l'éclatement de la vie sociale, que

\section{LA RITUALISATION}

LAISSE ENTENDRE

QU'UNE CERTAINE

\section{DOMESTICATION DE L'AUTRE}

EST PLAUSIBLE.

de rituels religieux proprement dits. Pensons par exemple aux rituels militaires, aux rituels alimentaires, aux rituels d'intoxication et de guérison, aux marquages corporels ${ }^{9}$ de toutes sortes, aux technoritualités, aux ritualités carnavalesques et aux raves ${ }^{10}$, sans compter les rituels mass-médiatiques de la téléréalité qui, littéralement, mettent en scène des processus de mort et de salut symboliques $^{11}$, pour ne nommer que les plus évidents. Tous les rituels, même ceux qui appartiennent d'office à des institutions religieuses, intègrent des composantes séculières. De même que les rituels appartenant à l'institution séculière du monde comportent généralement des dimensions religieuses, non seulement par l'invention de mythes qui leur sont propres, mais souvent sous forme d'emprunts plus ou moins admis aux traditions religieuses elles-mêmes. Il existe donc une dialectique du religieux et du séculier, propre aux phénomènes rituels, dont nous verrons d'autres exemples dans la prochaine section de ce texte.

\section{LES RITUELS FUNÉRAIRES DANS LA MODERNITÉ}

Un rituel est un dispositif symbolique qui vise l'intégration d'une communauté. En tirant cette définition minimale des réflexions précédentes, nous obtenons un guide pour mieux comprendre les transformations des rituels funéraires au $\mathrm{XX}^{\mathrm{e}}$ siècle.
Par dispositif symbolique, nous entendons un ensemble de mots, d'objets, de personnes, c'est-à-dire de réalités déjà dotées d'une valeur sociale reconnue, dont la circulation réglée produit des effets de sens. Si les rituels concernent les limites du sens, il est tout à fait normal que l'expérience de la mort en soit un terrain privilégié, dans toutes les civilisations. Les rituels funéraires, on le sait, sont même considérés par les paléontologues parmi les signes les plus révélateurs de l'apparition de l'humain sur terre. Insistons toutefois ici sur le terme dispositif: un rituel est un ensemble symbolique non seulement parce qu'il est ordonné comme un langage, mais parce qu'il est établi, stable et reconnu. Pour être efficace, il doit évidemment être facilement reconnaissable par ceux qu'il concerne.

En fait, les rituels appartiennent à l'institution culturelle des sociétés, c'est-à-dire au «système d'échange concret [qui y] rend compte des rapports sociaux en un lieu et un temps donné12 ». Organisation stable, un rituel ne se change pas facilement, et surtout pas au gré de la volonté de ses acteurs. Le changement risque d'en rendre les signes plus difficilement reconnaissables, même quand il vise à les rendre plus compréhensibles. Il peut alors avoir des effets pervers. Si les rituels «jettent des ponts sur les rivières d'angoisse qui bouillonnent en nous $^{13}$ » à cause de l'expérience de la rupture et du manque, leur efficacité tient bien moins à leur compréhension - nécessairement relative, puisque comprendre, c'est s'inscrire dans la relativité du langage - qu'à la reconnaissance automatique de leur dispositif. C'est ainsi que, même en langue morte comme on les pratiquait autrefois, ils pouvaient s'avérer intégratifs. Ils faisaient alors simplement appel à une sacralité échappant aux acteurs individuels mais congruente à leur communauté naturelle. Il est souvent arrivé que leur transposition en langue vernaculaire, quand on l'a tentée, les ait ouverts aux ambiguïtés du langage et, par là, aux évaluations subjectives ${ }^{14}$. Sans éducation adéquate, le changement dans les rituels peut s'avérer dévastateur des communautés qui pensent s'en soutenir, parce qu'il rouvre la porte à l'angoisse qui jusque-là pouvait sembler contenue, devant les incertitudes du sens.

Cette stabilité des rituels ne signifie pourtant pas qu'ils ne peuvent changer. À la manière d'une langue vivante, chaque fois qu'ils sont exécutés ${ }^{15}$, les rituels sont au contraire porteurs de nouvelles appropriations de sens. Leurs transformations ne peuvent cependant être le seul effet de la volonté d'acteurs particuliers, fussent-ils établis en autorité. Les rituels témoignent d'une dynamique communautaire. Ils ont comme objectif l'intégration d'une commu- 
nauté et dépendent aussi, pour signifier, de l'état actuel de cette communauté. Or cette dynamique n'appartient à personne en particulier. La prendre en charge au nom d'une prétendue maîtrise (au nom du savoir, de l'autorité, voire de la saine doctrine) risque de les rendre dysfonctionnels: s'établit alors une disjonction entre la volonté explicitée et la dynamique implicite qui répond à d'autres lois, à d'autres impulsions. Bref, quand les rituels se transforment, comme ils l'ont fait au XX $\mathrm{X}^{\mathrm{e}}$ siècle, c'est surtout parce que les dynamiques de régulation globale de la société à laquelle ils appartiennent se trouvent elles-mêmes en transformation.

Les rituels accompagnant l'expérience de la mort sont des témoins privilégiés de cette réalité. $\mathrm{Au} \mathrm{XX} \mathrm{XX}^{\mathrm{e}}$ siècle, ils ont connu des transformations radicales à la mesure des changements socioculturels et complètement indépendantes des capacités de contrôle des individus et des groupes constitués, fussent-ils les Églises qui présidaient traditionnellement à leurs destinées. L'éminent historien Philippe Ariès parle à ce propos de «la mort inversée $\mathrm{e}^{16} »$. Or cette inversion, par les forces qui la déterminent, correspond exactement à ce qui se passe dans d'autres secteurs de la société, comme la vie affective, la sexualité, les loisirs, le travail lui-même. Alors que toutes ces activités étaient autrefois réglées par des impératifs de la communauté dite naturelle, dont les principales figures étaient celles de la famille, du village et de la nation, elles sont aujourd'hui renvoyées à la responsabilité des individus. Ceux-ci les gèrent dans les limites de leur rationalité, toujours redevable, en dernière analyse, de la logique régulatrice du marché.

Évidemment, dans la mesure où l'enjeu des rituels est la communitas, un tel changement dans la source de leur régulation comporte des conséquences majeures. La logique des marchés, au-delà de la compétition et de la concurrence qui en sont les traits les plus visibles, vise essentiellement à articuler des supposés besoins à des satisfactions plausibles et vérifiables. Elle est donc tout naturellement réfractaire à la communauté, non pas par principe, mais parce que son dynamisme exige qu'elle soit d'abord sensible à la diversité des besoins des individus, pour y accorder son offre. Les communautés naturelles assuraient leur équilibre en harmonisant les différences entre leurs membres et elles se prévalaient pour cela des modèles, des idéaux et des normes pratiques auxquels elles appelaient chacun à se conformer. Le défaut de conformité entraînait l'exclusion. Les marchés assurent leur dynamisme - et par là leur progrès - en favorisant la concurrence de chacun contre tous. Non seulement cela rend-il cruciale la position de l'individu, mais cela en fait le principal acteur, celui qu'il faut à la fois promouvoir et contrôler, de façon à ce que ses comportements acquièrent le maximum de pertinence, pour s'inscrire dans une évaluation adéquate de ses besoins et des possibilités de satisfaction qui lui sont offertes.

des dernières volontés du mourant. Espérée sereine et sans violence, la «bonne mort» supposait ainsi un état de conscience harmonieux quant aux rapports du sujet avec sa communauté, et la présence de cette dernière était requise pour en attester. Institution d'encadrement de la communauté naturelle, la religion venait également dire le sens au chevet du moribond. Accompagner un agonisant, dans ce contexte, signifiait la plupart du temps d'abord prier avec lui, tant que cela était possible et ensuite prier pour lui, de façon à lui assurer les meilleures conditions possibles de passage dans l'au-delà.

Le contraste est évidemment frappant quand on considère les conjonctures contemporaines de la mort. On meurt généralement aujourd'hui d'accident: accident de la route, accident d'une histoire personnelle dans l'impasse ou accident d'une rationalité médicale arrivée à ses limites. L'environnement rationnel offert par les institutions de santé, voire celles qui proposent des soins palliatifs, ne pèche pourtant pas par manque d'empathie. Au contraire, en s'efforçant d'enlever la souffrance, on entend bien y optimaliser les conditions de sérénité de la bonne mort. Mais en même temps, par son besoin de contrôler les facteurs de cette sérénité, il fait du mourir un acte privé, intime, dont l'accompagnement, quand il existe - quand l'accident n'est pas trop brutal - se réduit à quelques proches rigoureusement sélectionnés, sinon à quelques spécialistes, ceux $\mathrm{du}$ cheminement spirituel s'associant au mieux, quand ils sont requis, avec ceux du contrôle de la souffrance.

On peut, dès qu'on y porte un minimum d'attention, discerner les traits des ritualités qui s'installent au chevet du malade. Il s'agit d'abord, essentiellement de technoritualités, dont les dispositifs symboliques visent avant tout l'efficacité des soins et, en consé-

Voilà, rapidement esquissé, le contexte des rituels funéraires contemporains. Cela dit, il faut, aujourd'hui comme autrefois, considérer trois champs de pratiques rituelles autour de l'expérience de la mort: l'acte de mourir lui-même, la disposition des restes et les discours sur l'au-delà ${ }^{17}$.

Dans les sociétés traditionnelles, l'acte de mourir supposait un ensemble de gestes publics, souvent ostentatoires, accompagnés de discours éminemment ritualisés mais dont on pouvait aussi attendre des paroles signifiantes, susceptibles de marquer ceux qui devaient survivre, notamment dans l'expression e, produisent la sérénité. Il s'agit, de plus, de ritualités essentiellement séculières. Quand elles donnent place aux signifiants religieux, c'est en marge de leur contrôle rationnel et, le plus souvent, dans la mesure où elles peuvent instrumentaliser ces signifiants. Cela ne signifie cependant pas que tout enjeu communautaire en soit absent. Bien au contraire. La communauté qui se dessine au chevet du malade est cependant surtout une communauté thérapeutique, ou mieux une communauté des affectés par la conjoncture de soins, intégrant soignants et proches. Il ne faut pas se le cacher, en 
effet, le caractère inéluctable de la mort qui approche affecte les soignants : leur raison d'être fondamentale, servir la vie, y est toujours d'une façon ou d'une autre mise en cause. Il arrive un moment où les soins deviennent futiles ${ }^{18}$, en termes de possible guérison, et doivent donc trouver d'autres raisons d'être. Les technoritualités de l'accompagnement visent le dépassement de ce trauma. Soignants et proches, a priori étrangers les uns aux autres, sont ici réunis par la violence du réel qui s'impose. Les processus rituels concernent évidemment la communauté qu'ils sont encore appelés à réaliser quand il faut prendre certaines décisions éthiques concernant, par exemple, la prolongation ou non des soins.

La communauté des affectés est bien, on le voit, une réalité concrète, quoique jamais définitive. Deux films récents, au Québec, ont mis en scène cette recomposition communautaire, dans un contexte ou la mort annoncée laisse du temps aux divers affectés pour le réaménagement de leurs relations. Dans Les invasions barbares et La vie avec mon père ${ }^{19}$, la ritualisation séculière de l'approche de la mort donne lieu à la remise en chantier des rapports amicaux et filiaux à travers des retrouvailles, des repas partagés, des remémorations, de l'entraide et de la connivence entre soignants et proches, dans des gestes plus ou moins recherchés mais finalement efficaces. Les rapports humains sont a priori éclatés, les personnes étant devenues plus ou moins étrangères les unes aux autres, mais chacun en sort transformé. Les amis, les fils, les filles, les anciens amants sont renvoyés à la suite de leur histoire propre, mais c'est après avoir partagé une expérience du manque qui a altéré leur vision du monde. Il s'agit bien là d'un enjeu communautaire: il se résout par des solidarités nouvelles, auxquelles aucun lendemain n'est assuré et qui n'atteindront vraisemblablement jamais le degré d'institutionnalisation des communautés naturelles d'autrefois, mais qui concernent le sens dans lequel chacun va engager sa survie.

Le deuxième champ de pratiques, celui du traitement du cadavre, a également été marqué de transformations profondes au cours du dernier siècle. Il est très largement devenu, lui aussi, un champ de technoritualités séculières, tout d'abord en ce qui concerne la préparation du corps. Le toilettage, qui était la responsabilité des proches du défunt, en particulier des femmes, est désormais remplacé par un travail professionnel, sous l'enseigne de la thanatopraxie, qui prend en charge les dispositions légales, sanitaires et esthétiques dont se compose le processus funéraire. Rapidement, en Amérique, les salons funéraires ont remplacé les demeures privées pour l'exposition

\section{LE RITUEL}

\section{VISE À DOMESTIQUER}

\section{LA JOUISSANCE DE L'AUTRE.}

du corps, adaptant ainsi le rituel aux modes de vie urbains. Pendant quelques décennies, ils ont pris une place majeure dans le dispositif global, place qui semble aujourd'hui encore en pleine transformation, de plus en plus de personnes choisissant la disposition directe, sans présentation du corps, ou encore la présentation de l'urne contenant les cendres ou du cercueil fermé, voire d'une photographie du défunt. Sans même rien de tout cela, on peut aussi choisir de simplement accueillir les condoléances dans l'église, avant la cérémonie religieuse.

Quoi qu'il en soit, même en contexte d'extrême restriction des espaces alloués à la présentation des restes, on peut encore voir s'y dessiner l'enjeu de la recomposition communautaire. Que fait-on, en effet, au salon funéraire, à l'arrière de l'église, voire plus encore à l'occasion de la réception - rappel des antiques banquets funéraires - qui de plus en plus souvent suit la cérémonie des funérailles proprement dite? Essentiellement, on parle, on échange des paroles de condoléances, de soutien, de souvenir, paroles toujours à la fois ritualisées et personnalisées. La présence du cadavre, fût-elle purement symbolique, est l'occasion d'un rappel de sa vie, des expériences vécues de son vivant, et, bien sûr, comme tout récit mémoriel, d'une recomposition de son histoire, de façon à lui donner une épaisseur appréciable. Dans ce processus discursif se dessinent d'ailleurs des autorités: l'époux ou l'épouse, les fils et filles, les proches, selon des stratégies complexes d'accréditation (que les directeurs de funérailles gèrent au mieux, parfois sur le tas). Tout le monde n'est pas écouté de la même façon. Tout le monde ne tient pas le même discours. Une discrimination s'effectue en fonction des réseaux affectifs en recomposition, où des solidarités, anciennes ou nouvelles, sédimentées ou en gestation, s'affirment et parfois se contredisent ${ }^{20}$.

Théoriquement, quand il y a cérémonie religieuse à l'église, c'est cette «communauté» en recomposition qui s'y présente,

\section{LE RITUEL}

FAIT DE L'AUTRE SAUVAGE LE COLLABORATEUR D'UNE HISTOIRE SENSÉE. avec ses richesses, ses incertitudes et ses imbroglios. Quand les rituels religieux avaient affaire à des communautés naturelles, ils pouvaient assez facilement en accueillir la réalité, puisqu'elle était connue de tous, établie et instituée. Ce n'est évidemment plus le cas. Aussi les autorités religieuses reçoivent-elles de plus en plus souvent des demandes de rituels personnalisés qui compliquent considérablement leur vie. Les dispositifs rituels, pour s'exercer efficacement - lancer des ponts sur l'angoisse qui accompagne l'expérience du non-sens doivent être idéalement sans équivoque. Il est de toute évidence extrêmement difficile de contrôler des paroles - par exemple d'hommage au défunt - qui peuvent, à la limite, tourner au panégyrique sans pudeur ou s'avérer de subtils règlements de compte entre les survivants. L'aptitude des dispositifs rituels à concourir à la recomposition communautaire est alors singulièrement mise en cause.

Le troisième champ de pratiques, celui que nous appelons le champ des discours sur l'au-delà, subit par ailleurs les effets de l'éclatement du monde des croyances en Occident. Pour bien des personnes, il est évident que les propositions traditionnelles de sens concernant le salut, la résurrection, le paradis, l'enfer, sont devenues plus ou moins langue de bois. Pourtant, quand on observe certains discours qui se veulent proprement séculiers, on ne cesse d'être surpris de la persistance de références à la vie après la vie qui évoquent un au-delà bien concret. Les marqueurs symboliques, certes, en sont parfois incertains, mais, à l'instar d'occurrences fréquemment trouvées également dans les notices nécrologiques, ils ne dédaignent pas de s'adresser au disparu comme s'il était toujours là, dans une proximité à la fois insaisissable et tenace, opaque et lumineuse. La métaphore de la présence s'y file à un point tel que le disparu «nous entend et nous regarde», "qu'il nous accompagne maintenant». "Déjà étoile sur la terre, Marie-Soleil est désormais une étoile dans le ciel pour éclairer notre $v^{2} \mathrm{e}^{21} » \ldots$

Est-ce là simple persistance du religieux? La vie des signes dans la vie sociale n'est pas si simple qu'on puisse conclure abruptement. Le monde séculier, qui s'est constitué historiquement au sein du monde religieux, n'incarne que rarement «un pur dehors du religieux, sans lien ni dette envers lui ${ }^{22} »$. Acculé à sa propre expérience des limites, aux confins de sa rationalité, il lui arrive de n'avoir d'autre choix que de reproduire les "virtualités fondamentales» du religieux, en risquant à son tour un discours sur l'Autre, à sa façon. Une pudeur bien compréhensible, sinon le rejet explicite des traditions, l'empêche d'appeler « religieux », selon les codes traditionnels, ce qui tient 
pourtant lieu d'assurance contre le chaos et répercute les vieilles fonctions du religieux... L'expérience de la mort, il faut bien le reconnaître, représente un point de butée de la sécularité.

\section{LA COMMUNAUTÉ EN JEU}

Mais quelle est donc cette communauté que nous voyons poindre dans l'ombre des rituels, y compris là où ils présentent un visage technique et séculier?

Il ne s'agit certes pas de la communauté naturelle qui présidait autrefois aux rituels funéraires. La modernité, on le sait, l'a irrémédiablement dispersée. Et même si on en réactualise les signifiants par une surenchère de rappels et de références, comme on l'a vu dans les églises au cours des dernières décennies, cela ne la fait pas revivre. $\mathrm{Au}$ contraire, cela accentue, bien souvent, l'impression de langue de bois chez ceux qui subissent ce vocabulaire alors qu'ils s'en savent étrangers. Une véritable impasse peut s'installer quant la réalité - éclatée - de l'assemblée ne correspond pas à la surenchère communautaire du discours. Tout rituel à cet égard, engage des exigences de vérité qu'il faudrait apprendre à reconnaître ${ }^{23}$.

L'assemblée qui se rassemble aux funérailles, même si elle donne une place privilégiée aux proches, n'est donc pas assimilable à la communauté naturelle du village ou de la famille dont la tradition nous a légué mémoire. Elle n'est pas non plus une communauté affective proprement dite. Chacun, certes, peut participer à des réseaux de liens affectifs à l'intérieur de l'assemblée, mais rien n'en garantit l'unité. Dans bien des cas, on se trouve même en présence de réseaux concurrentiels, sinon antagonistes. Les familles sont aujourd'hui largement recomposées et même si ses formules complexes sont de plus en plus souvent ouvertement admises et évoquées dans les rituels, on ne peut pas parler de ces recompositions au même titre qu'on parlait autrefois de communautés.

La communauté, en situation de société contemporaine, ne peut être non plus une communauté de l'imaginaire. On sait à ce propos combien les croyances sont désormais éclatées, non pas d'abord à cause des diversités ethniques et culturelles, mais dans la mesure où leur individualisation est tributaire des lois du marché. Chacun, quelle que soit son origine, même quand il continue à se référer à des traditions, adapte ses représentations du sens en fonction des besoins qu'il ressent et en puisant dans l'offre qui lui est faite. Or, médiatisée massivement, cette offre est désormais pléthorique et ne connaît pas de frontières. Dans une société dont les régulations dominantes sont des régulations de marché, il est tout à fait logique que ces dernières pénètrent également les

DANS UNE SOCIÉTÉ DONT LES RÉGULATIONS DOMINANTES

SONT DES RÉGULATIONS DE MARCHÉ, IL EST TOUT À FAIT LOGIQUE

QUE CES DERNIÈRES PÉNÈTRENT ÉGALEMENT LES RITUELS FUNÉRAIRES,

DANS UNE RECOMPOSITION DE L'IMAGINAIRE CROYANT QUI N'EST

NI PROGRAMMÉE NI VOULUE PAR UNE AUTORITÉ QUELCONQUE, MAIS QUI TEND À S'ACCORDER À LA LOGIQUE DES BESOINS/SATISFACTIONS.

rituels funéraires, dans une recomposition de l'imaginaire croyant qui n'est ni programmée ni voulue par une autorité quelconque, mais qui tend à s'accorder à la logique des besoins/satisfactions. Le manque mis à nu dans l'existence, dans la proximité de la mort, crée un besoin de croyance. Le marché tend à y répondre, provoquant du même coup un éclatement de l'univers des croyances ${ }^{24}$.

La logique de marché, avons-nous avancé, est réfractaire à la communauté. Elle se déploie d'autant mieux qu'elle s'adresse à des individus, supposés libres, non entravés par des solidarités qui pourraient affaiblir leur performance et biaiser leur jugement, qu'elles soient familiales, amicales, confessionnelles ou même nationales. Mais si les assemblées qui se réunissent à l'occasion des funérailles sont éclatées de tous points de vue, de quoi est-on en train de parler, quand on évoque leur enjeu communautaire?

Signalons d'abord que la pluralité en ellemême n'a rien de contradictoire par rapport aux dynamiques rituelles telles que nous les avons présentées. Bien au contraire, si ces dernières visent une recomposition communautaire, logiquement elles doivent bien partir d'une situation de dispersion, sinon de décomposition comme cela peut être le cas de certaines traditions.

À ce propos, faisant état de la crise contemporaine de la parole dans les célébrations eucharistiques, l'ancien supérieur général des Dominicains, Timothy Radcliffe, esquissait récemment la structure de ces célébrations à partir de la scène primitive du christianisme que représente le dernier repas de Jésus avec ses disciples, événement qui prend place juste avant que le processus de sa mise à mort soit amorcé 25 . Dans ce dernier repas, montre-t-il, se présentent «trois moments» dont le premier, précisément, est celui de l'accueil de la dispersion, c'est-à-dire des "difficultés et désarrois personnels » des disciples, traumatisés par ce qu'ils voient venir. Le deuxième est celui de la recomposition communautaire proprement dite, dans le partage du pain, du vin et de la parole, et le troisième celui de l'ouverture à l'autre, quand chacun des participants est appelé à devenir responsable de cette expérience de solidarité vécue et accepte de la re-présenter dans le monde. Radcliffe qualifie l'événement de happening, parce qu'il inaugure, en chacun, une conscience nouvelle, selon le sens plein du mot expérience ${ }^{26}$. La sacramentalité des gestes et des paroles, dans son vocabulaire théologique, repose elle-même sur cette capacité de contribuer à la communauté, c'est-à-dire à une expérience solidaire du monde, de la vie, du manque, du sens.

La solidarité humaine ne se construit pas sur les prétendues similitudes entre les humains, mais bien sur l'accueil de leurs différences.

Ainsi, ce que nous appelons communauté ne peut plus être appréhendé comme une réalité empirique matérielle, stable et objective, bref, instituée. La communauté, dans la dispersion sociale, à l'instar du sens dans la pratique du langage, est une réalité en gestation, un processus, un élan du désir. On ne la rencontre, de fait, que dans un mouvement, dans le travail de dépassement de la dispersion qui est toujours à recommencer parce que le réel, c'est la dispersion, comme le rappelle la mort, sans complaisance. La communauté, pour le dire autrement, n'est pas un état de fait mais un fruit escompté du désir humain. Elle ne peut se réduire à un acquis, ni à un capital sur lequel on pourrait compter. Sa nature est dynamique. Elle se manifeste dans le travail de renouvellement des solidarités humaines, dans le travail de symbolisation de l'utopie que celles-ci peuvent représenter, parce qu'elles ne sont jamais acquise. Une telle communauté d'intention n'est jamais soluble dans les relations de marché mais, du même coup, elle représente ce en quoi la vie s'humanise, quand à partir des différences sexuelles, sociales, raciales, à partir des histoires de chacun, bref sans renier la dispersion mais en l'assumant, une expérience signifie à chacun sa participation au statut d'humain, singulier mais solidaire.

Or c'est là précisément le travail du rite, tel qu'il devient indispensable quand s'impose l'expérience de la mort. 
Sur-vivre: tel est en fait l'enjeu véritable de ce travail. Le sens originel du mot ne concerne ni la supposée «vie après la vie» des ésotérismes contemporains, ni la «vie éternelle» proposée par les traditions monothéistes. Il concerne le fait de continuer de vivre malgré et dans l'expérience de la mort, continuer de vivre en assumant la perte, le deuil, la rupture, la disparition de l'autre et l'inconnu de son propre destin, aux échéances toujours trop proches. Vivre dans la solidarité avec tous les autres, ceux qui vont mourir, pour que le sens, la communauté, la solidarité ne représentent pas de simples utopies mais deviennent des choix et des pratiques actualisés. En effet, «la vie est survie»; "c'est l'affirmation d'un vivant qui préfère le vivre et donc le survivre à la mort, car la survie, ce n'est pas simplement ce qui reste, c'est la vie la plus intense possible $^{27}$ ». Et cette survie suppose, dans le quotidien, un courage d'être qui doit sans cesse être renouvelé, jusqu'à l'héroïsme. C'est à ce point, très précis, que le rituel jette des ponts sur les rivières d'angoisse qui coulent en nous. Il propose des espaces symboliques pour ce choix de l'humain, ce choix toujours aléatoire par lequel chacun est renvoyé à sa singularité, à sa condition de sujet assujetti et désirant. La communauté qu'il intègre ne peut être qu'une communauté en changement, friable et par cela même confrontée à l'angoisse de sa perte, toujours imminente. Mais cette communauté du désir est la seule qui, aujourd'hui, puisse faire front au réel.

Ainsi trouve-t-on un sens plein de la communitas évoquée par Victor Turner. Élan, travail (ce qui est le sens étymologique du mot liturgie, en l'occurrence), actualisation d'un désir inachevé de solidarité, elle ne peut qu'être orientée vers l'à-venir. La solidarité des humains, son enjeu, n'est pas réfractaire à la dispersion qui est sa condition de possibilité, mais est bien réfractaire, plutôt, à tout caractère définitif qu'on voudrait lui donner.

$$
* * *
$$

«L'individu a horreur de la mort parce que cette dernière détruit sa singularité. [...À] partir du moment où l'individu devient une valeur cardinale, avec ses cultes inhérents (jeunesse, fonctionnalité, indépendance), la mort se dresse comme déchéance et échec insupportable», écrit encore Luce Des Aulniers ${ }^{28}$. Sans doute est-ce la raison la plus fondamentale pour laquelle on a tendance à la cacher - alors même que sa mise en spectacle est constante dans les médias. La mort fait honte, parce qu'elle symbolise notre impuissance. Mais cette honte elle-même est porteuse de mort. L'humain, être de désir, est un affamé de sens et «[s]'il ne parvient pas à vaincre sa honte, l'affamé meurt ${ }^{29} »$.
Devant l'expérience du manque, il ne lui reste en réalité que deux choix. Soit jouer aux apprentis sorciers dans une fuite en avant technologique - vaincre la mort devenue idéologie dominante dans les sociétés dopées par le «progrès» soutenu de leurs innovations, au point qu'elles en viennent à appeler développement, dans un rêve qui tourne au cauchemar, le mépris de la vie dans l'adoration des choses ${ }^{30}$. Soit tenter de vivre avec le manque, dans la conscience la plus réaliste possible du tragique de la condition humaine, ce tragique dont les accents ne sont pas moins réels du fait qu'ils sont muselés. Ritualiser le tragique au cœur de l'humain, c'est foncièrement tenter de vivre avec le manque et assumer, par le fait même, la condition humaine. La communauté en processus qui se dessine alors, est appelée comme un lieu, ou plutôt un acte de sens, provisoire mais signifiant, dans lequel les sujets, irréductibles dans la singularité de leur expérience, sont appelés à trouver un espace. Ce que nous avons appelé survivre.

Ce nécessaire travail des rituels est exigeant, puisqu'il consiste à parcourir les frontières où se marquent la présence et l'absence de la vie. Il ne peut se présenter que dans un parcours des limites, autrement dit, une aventure dans laquelle le sujet est appelé à advenir à la conscience de son être, coupé de ses rêves d'éternité. Expérience liminale, il convie à se tenir sous la règle de la limite: sub limen, c'est-à-dire là même où se profile le sublime. Qu'est-ce à dire? Là où le sujet s'abandonne au réel, tout en se manifestant comme désir de vivre, acte de survie, là où il s'agit de "s'abandonner sans réserve à ce qui vous emporte, comme on se confierait à son pire ennemi, [...] tandis que disparaissent limites, frontières et cloisonnements ${ }^{31}$ ». Élan, dépassement, le sens qui refuse le trait définitif et s'inscrit plutôt comme acte de désir ne peut effectivement se résoudre que dans un appel au sublime.

Dans ce travail de sublimation, la parole personnelle doit toujours être présente, puisque c'est par elle que se dit la singularité et la vérité de l'être en quête de solidarité. Mais la parole n'est pas non plus suffisante. Dans la mesure où elle suppose un langage institué, elle subit les limites de son institution. On la voit souvent devenir banale et arrêter l'élan constitutif du sens, plutôt que le nourrir. D'autres formes de symbolisation doivent alors prendre la relève, des formes propres au champ de l'esthétique qui, telle la musique, font entendre l'harmonie dans le chaos et inscrivent au cœur de l'épreuve une joie venue d'ailleurs. Un rituel est aussi une forme d'art qui peut ouvrir des chemins inattendus, modulés par des styles, où le sujet est appelé au dépassement. L'é-motion ne lui est pas étrangère: elle est une autre forme de mise en mouvement. Les grandes liturgies, comme travail de dépassement, se jugent ainsi à leur beauté. Elles culminent quand, échouant à nommer, définir, décrire, échouant à réduire le monde à l'ordre établi des langages - ordre pourtant nécessaire elles ouvrent des espaces renouvelés pour l'inscription de la survie, elles supportent les élans des sujets vers un supplément d'être. La beauté partagée est sans doute le lieu ultime du sens et de la communauté, l'antidote de la banalité et de l'isolement, quand l'humain achoppe aux bornes de son histoire.

\section{Notes}

1. Jean-Pierre Vernant (2004), La traversée des frontières, Paris, Seuil, 2004, 196 p.

2. Cornelius Castoriadis (1997), Fait et à faire, tome V des Carrefours du labyrinthe, Paris, Seuil, cité par Robert Redeker, "Contre le conformisme généralisé», Le Monde diplomatique, août 1997, p. 24.

3. Luce Des Aulniers (1984), «Le cœur déchiré de l'existence", dans Raymond Lemieux et Réginald Richard (dir.), Survivre... la religion et la mort, Montréal, Bellarmin, p. 255-274.

4. Victor W. Turner (1969), The Ritual Process. Structure and Anti-Structure, Chicago, Aldine Publishing Company, 213 p.

5. Catherine Bell (1992), Ritual Theory, Ritual Practices, New York, Oxford University Press, 1992.

6. Victor W. Turner et Edith Turner (1978), Images and Pilgrimages in Christian Culture Anthropological Perspectives, New York, Columbia University Press, $281 \mathrm{p}$.

7. Bernardi, Ermano, «L'apport de V. W. Turner à une anthropologie du pèlerinage », $L a$ Maison Dieu, nº 170, 1987, p. 80.

\section{Ibidem.}

9. Voir à ce propos Religiologiques, 16, automne 1977: Rites sauvages, numéro coordonné par Denis Jeffrey, Guy Ménard et Jacques Pierre, Département de sciences religieuses de l'Université du Québec à Montréal, 211 p.

10. Religiologiques, vol. 24, 2002; Technoritualités. Religiosités rave, sous la direction de François Gauthier et Guy Ménard, Montréal, Université du Québec à Montréal, 288 p.

11.Jean-Pierre Desaulniers, "Loft Story, ou la dernière scène », Le Devoir, 17 octobre 2003, p. A10.

12. René Lourau (1969), L'instituant contre l'institué, Paris, Anthropos, p. 172-175.

13. Cf. Denis Jeffrey, Ritualité et postmodernité. Pour une éthique de la différence, thèse de doctorat en sciences religieuses, Université du Québec à Montréal, mars 1993, p. 127.

14. Voir Raymond Lemieux, «Retrouver le sens $\mathrm{du}$ rituel. Enjeux des pratiques et des rites funéraires », Frontières, vol. 4, n 1 , printempsété 1991, p. 6-12. 
15. Voir chez Ferdinand de Saussure, la conception de la parole comme «exécution» de la langue, cette dernière étant l'institution élémentaire qui, chez l'humain, permet l'actualisation de nouvelles subjectivités chaque fois que quelqu'un parle. Cours de linguistique générale, Paris, Payot, 1975 (première édition: 1925), $510 \mathrm{p}$.

16. Philippe Ariès, "La mort inversée. Le changement des attitudes devant la mort dans les sociétés Occidentales", dans Essais sur l'histoire de la mort en Occident du Moyen Age à nos jours, Paris, Éditions du Seuil, coll. «Points» H31, 1975, p. 177-210.

17. L'énoncé de ces champs provient d'une recherche que nous avons menée, à Québec, au début des années 1980. Voir Raymond Lemieux, "Pratique de la mort et production sociale», Anthropologie et sociétés, vol. 6, $\mathrm{n}^{\circ} 3,1982$, p. 25-44, de même que le collectif sous la direction de Raymond Lemieux et Réginald Richard, Survivre... La religion et la mort, Montréal, Bellarmin, "Les Cahiers de recherches en sciences de la religion », vol. 6 , 1984, 285 p.

18. Robert Haliday, «Medical Futility and the Social Context», Journal of Medical Ethics, 1997, 23, p. 148-151.
19. Denys Arcand (2003) pour le premier et Sébastien Rose et Stéfanie Lasnier (2005) pour le second. Au sujet du film d'Arcand, on pourra lire mon article "L'accompagnement spirituel au temps des Invasions barbares», Cahiers de spiritualité ignacienne, septembre-décembre 2004; Parler la maladie. L'accompagment des malades, $\mathrm{n}^{\circ} 111$, p. 9-20.

20. Voir Raymond Lemieux, "Pratique de la mort et production sociale», Anthropologie et sociétés, vol. 6, n 3, 1982, 25-44.

21. Cf. Raymond Lemieux, «Des fumérailles laïques à Montréal: Marie-Soleil Tougas», dans Bernard Kaempf (dir.), Rites et ritualités. Actes du congrès de théologie pratique de Strasbourg, Paris, Cerf, Lumen Vitæ, Novalis, 2000, 261-267.

22. Marcel Gauchet (1985), Le désenchantement du monde. Une histoire politique de la religion, Paris, Gallimard NRF, p. 68.

23. Nous avons évoqué plus longuement ailleurs cette aporie, encore fréquente dans les paroisses qui se veulent «communautés chrétiennes»: "La liturgie en quête de vérité», Liturgie, foi et culture », vol. $38, \mathrm{n}^{\circ} 177$, printemps 2004, 23-32.
24. Voir: «Les croyances des Québécois», Cahiers St-Charles, 12, Sherbrooke, février 2003, 37-56. www.ftsr.ulaval.ca.

25. «Prédication: sortir de l'ennui!», Études, janvier 2003, 63-74.

26. Comme le propose notamment Hans-Georg Gadamer dans Vérité et méthode. Les grandes lignes d'une herméneutique philosophique, Paris, Seuil, coll. «L'ordre philosophique», 1996, p. 376-377.

27. Jacques Derrida, «Je suis en guerre contre moi-même», propos recueillis par Jean Birnbaum, Le Monde, jeudi 19 août 2004

28. «Une violence occultée», Relations, no 496, novembre 2004: Sur les traces de la mort, p. 13.

29. Jean Ziegler (1995), L'empire de la honte, Paris, Fayard, 323p.

30. Voir Serge Latouche, Survivre au développement. De la décolonisation de l'imaginaire économique à la construction d'une société alternative, Paris, Mille et une nuits, 2004, 120 p.; Décoloniser l'imaginaire: la pensée créative contre l'économie de l'absurde, Paris, Parangon, L'Aventurine, 2003, 126 p.

31. Catherine Milot (2001), Abîmes ordinaires, Paris, Gallimard, coll. «NRF», p. 40.

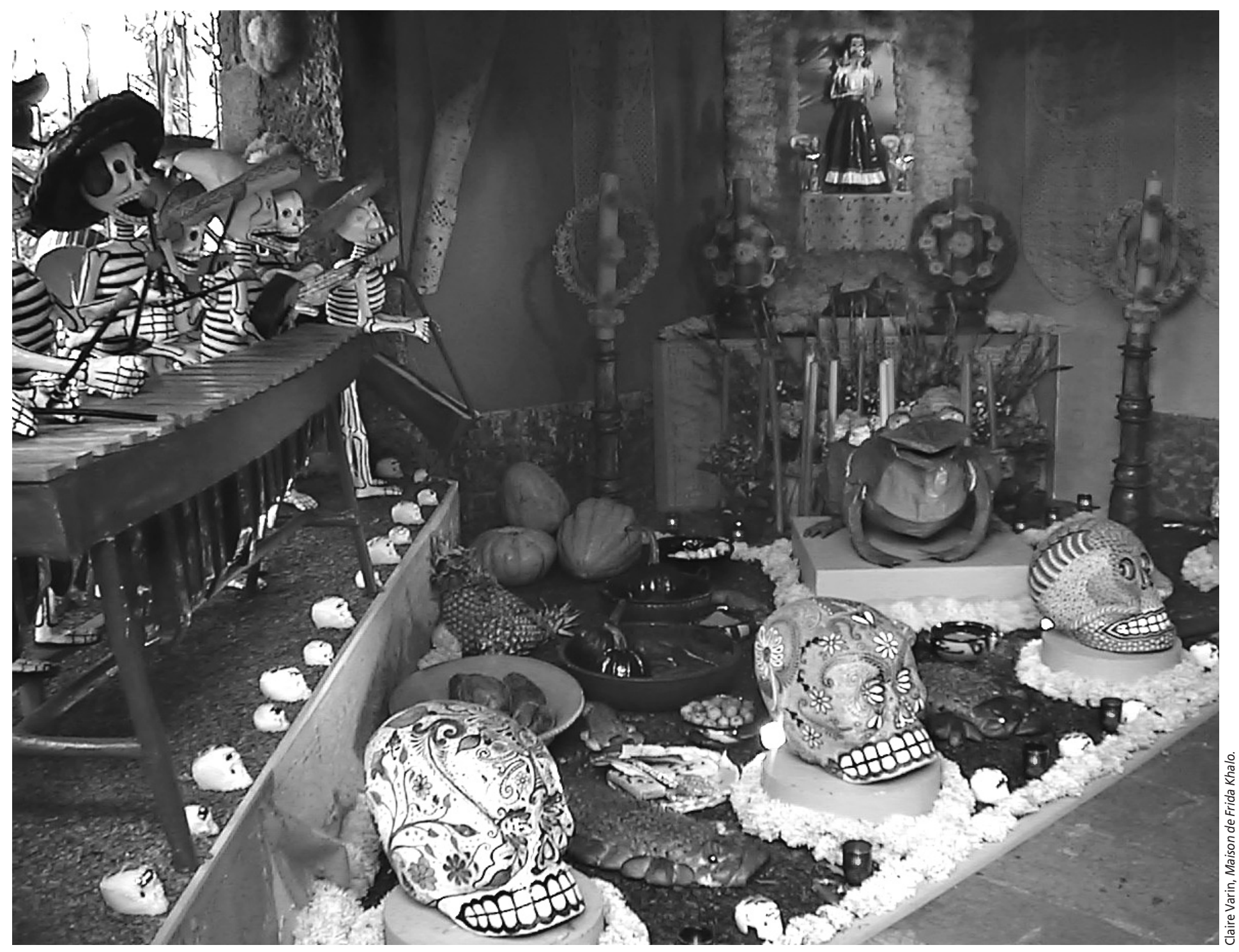

